\title{
OPTOFLUIDICS
}

\section{Air-tuned dye laser fits on a chip}

Given their broad wavelength tunability, dye lasers have historically played an important role in spectroscopy and other applications. Now, it looks like lab-on-a-chip systems may soon benefit from a miniature version that offers on-chip integration and a convenient pneumatic scheme for wavelength tuning. Wuzhou Song and Demetri Psaltis from the Swiss Federal Institute of Technology Lausanne now report an optofluidic dye laser that operates in the visible regime with emission that can be tuned through the air pressure applied to an integrated air-gap etalon (Appl. Phys. Lett. 96, 081101; 2010).

The laser chip is made by replica moulding of an elastomeric material called polydimethylsiloxane (PDMS). The design comprises a liquid core waveguide, $800 \mu \mathrm{m}$ long and $4 \mu \mathrm{m}$ wide, located between two microscale air chambers to form a laser cavity. One air chamber has an angled reflective interface and functions as a simple end mirror. The other, which features two parallel surfaces, functions as a low-finesse air-gap Fabry-Pérot etalon,
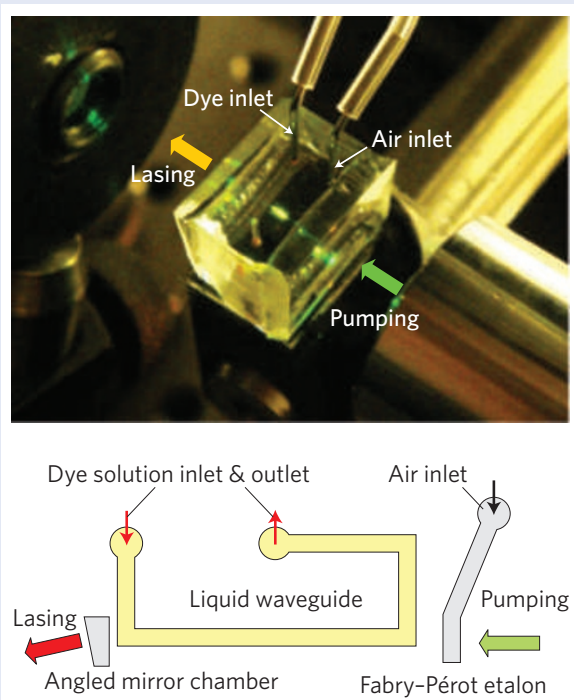

and is used to select the laser's desired wavelength of operation. The laser is longitudinally pumped through a series of low-power laser pulses. Its emission wavelength is tuned simply by using a pressure regulator to control the air pressure applied to the etalon's air-gap, which changes from its nominal width of $12 \mu \mathrm{m}$ as the applied pressure is varied from 2 to 30 psi.

The researchers used a mixture of two different dyes - rhodamine $6 \mathrm{G}$ and rhodamine $B$ - to improve the effective tuning range of the laser ( 581-595 nm). They also adopted a longitudinal multimode pumping scheme to enhance the pumping efficiency. The results of the experiment revealed a tuning range of $14 \mathrm{~nm}$ and a pumping threshold of $1.6 \mu \mathrm{J}$ per pulse. A laser emission wavelength of $588.4 \mathrm{~nm}$ with a $3 \mathrm{~nm}$ linewidth was also obtained, and it was found that the air refractive index within the air-gap etalon increased at a rate of $2 \times 10^{-5}$ per psi. The researchers explain that pneumatic tuning has an important advantage over hydraulic tuning: a much faster response and tuning time due to the much lower viscosity. They also say that the design is stable against thermal fluctuations as the emission wavelength can be 'locked' by the air regulator.
SONIA SHAHI

\section{Sharper focus by random scattering}

Contrary to intuition, a disordered scattering medium can be exploited to improve, rather than deteriorate, the focusing resolution of a lens.

\section{Mathias Fink}

U nderstanding and controlling wave propagation in a random multiplescattering environment is a subject of great interest in a wide variety of domains ranging from solid-state physics and optics through to microwave, acoustics and seismology. Multiple scattering in a complex medium is usually considered as an inevitable perturbation and often a nuisance because it distorts an incident wavefront so strongly that its spatial coherence is lost after it propagates over a certain distance. In the realm of optics, at the spatial level it gives rise to the wellknown 'speckle' interference patterns. At the temporal level, a short pulse entering a random scattering medium can greatly spread over time because of the many possible paths that waves can take before leaving the medium. However, recent findings from Ivo Vellekoop and colleagues might change the way we look at scattering. Reporting in Nature Photonics, the researchers show that, in defiance of common wisdom, disordered scattering can be used to achieve a sharper focus point up to 10 times smaller than the diffraction limit of the lens being used ${ }^{1}$.

Multiple scattering occurs, for example, in biological tissues (optically), in granular or polycrystalline media (acoustically), in a forest (microwave radar) and in cities (where buildings affect wireless telecommunications). In seismology, the 'coda', which is the long signal arriving after the ballistic signal propagating in the direct path between the seismic source and the seismometer, also arises from multiple scattering. From an operational point of view, multiple scattering greatly limits the quality of imaging, focusing or communication. Focusing a wave through such a medium is quite a challenge. Although adaptive optics is a popular approach for correcting wavefront distortion induced by the environment, for example in astronomy and ophthalmic imaging, it cannot be 